\title{
Differentiation of mesenchymal stem cells to osteoblasts and chondrocytes: a focus on adenosine receptors
}

\section{Shannon H. Carroll ${ }^{1}$ and Katya Ravid ${ }^{2,3,4, *}$}

Skeletogenesis, either during development, post-injury or for maintenance, is a carefully coordinated process reliant on the appropriate differentiation of mesenchymal stem cells. Some well described, as well as a new regulator of this process (adenosine receptors), are alike in that they signal via cyclic-AMP (cAMP). This review highlights the known contribution of cAMP signalling to mesenchymal stem cell differentiation to osteoblasts and to chondrocytes. Focus has been given to how these regulators influence the commitment of the osteochondroprogenitor to these separate lineages.

The development of the skeletal system, as well as its repair, are dependent on the differentiation of both chondrocytes and osteoblasts from their common progenitor, the mesenchymal stem cell (MSC). There are two processes from which bone is formed. During intramembranous osteogenesis, bone is formed directly from MSC differentiation to osteoblasts. This process gives rise to flat bones, such as those of the skull and clavicle. In contrast, endochondral osteogensis requires MSC differentiation to chondrocytes and the formation of a cartilage template, which is followed by ossification by osteoblasts. This process is responsible for the formation of the long bones of the skeleton, and remains active in the growth plates of growing bones (reviewed in Refs 1,2$)$. Bone fracture repair recapitulates the events of skeletogenesis and is, therefore, used as an experimental model of bone formation (Ref. 3). These processes are regulated by paracrine actions between osteoblasts and chondrocytes, and thus, proper skeletogenesis requires precise control over the differential differentiation of the MSC to these lineages. Failure of chondrocyte or osteoblast precursors to proliferate and differentiate leads to various types of skeletal dysplasias, depending on the pathway involved (reviewed in Ref. 4).

\section{Skeletogenesis: an overview}

The osteochondroprogenitor

The majority of what is known about MSC differentiation along the skeletal lineage comes from embryology. Endochondral and intramembranous skeletogenesis begins with the proliferation and migration of mesenchymal

${ }^{1}$ Department of Biochemistry, Boston University School of Medicine, Boston, MA, USA

2Department of Medicine, Boston University School of Medicine, Boston, MA, USA

${ }^{3}$ Whitaker Cardiovascular Institute, Boston University School of Medicine, Boston, MA, USA

${ }^{4}$ The Evans Center for Interdisciplinary Biomedical Research, Boston University School of Medicine, Boston, MA, USA

${ }^{*}$ Corresponding author: Katya Ravid, Boston University School of Medicine, 700 Albany St Room W602, Boston, MA 02191, USA. E-mail: kravid@bu.edu 
cells to form condensations. These condensations are characterised as tightly packed cells that express specific condensation markers, and will determine the position, shape and size of the skeletal elements (Ref. 5). The transforming growth factor- $\beta$ and Hox family of molecules, derived from the epithelium, have been implicated in directing the formation of condensations. These molecules, along with the cell-cell and cell-matrix interactions that result from the compact nature of these cells, are thought to trigger MSC differentiation (Refs 5, 6).

As the osteochondroprogenitor is multipotent, lineage fate decisions must be made and maintained. The transcription factor runt-related transcription factor 2 (Runx2) is believed to determine osteoblast versus chondrocyte differentiation. Various studies have shown that upregulation of Runx2 induces osteoblastic differentiation, whereas its persistent expression in chondrocytes causes premature maturation and mineralisation (Ref. 7). Runx2 is expressed in prechondrogenic and preosteogenic condensations, but during early embryogenesis, outside signals regulate its expression (Ref. 5). Homeobox protein A2 (Hoxa-2) and Wnt (wingless-type mouse mammary tumor virus (MMTV) integration site) signalling have both been implicated in this process. Through specific inactivation in the developing limbs of mice, $\beta$ catenin, a downstream effector protein of Wnt signalling, was found to be essential to bone development as these embryos had impaired osteoblast differentiation (Ref. 8). Also, it was found that if $\beta$-catenin is activated, it upregulates Runx2 expression and MSCs differentiate to osteoblasts, rather than chondrocytes (Refs 8, 9, 10). These results implicate $\beta$-catenin in the determination of osteo- versus chondrogenesis.

\section{Chondrogenesis}

Chondrocytes are the first skeletal cells to arise, and their differentiation depends on the expression of the transcription factor sry (sex determining region Y)-box 9 (Sox9). The importance of Sox9 was discovered when a genetic mutation in humans was found to be the cause of campomelic dysplasia, characterised by severe cartilage abnormalities (Refs 11, 12). Genetic deletion of Sox9 in mice blocks chondrocyte differentiation at the point of mesenchymal condensation, indicating that it is necessary for the induction of chondrocyte differentiation (Ref. 13). In the prechondrogenic mesenchyme, Runx2 is expressed along with Sox9 (Ref. 14). However, studies show Sox9 to be dominant over Runx2 (Ref. 15) by promoting Runx2 protein degradation as well as inhibiting its transcriptional activity (Ref. 16). In addition, Nkx3.2 inhibits Runx2 transcription, enforcing the differentiation to chondrocytes (Ref. 17). Sox9, along with co-activators Sox 5 and Sox6, binds and activates promoters of chondrocytespecific genes. These include collagen $2 \alpha 1$ (Col2 $\alpha 1)$ and aggrecan (Ref. 5).

Also, important to chondrogenesis is chondrocyte maturation. This involves the transition of chondrocytes from proliferating, to nonproliferating and hypertrophic, and eventually apoptotic (Ref. 2). The expression of Sox9, along with the activation of CAMP response element binding protein (CREB) and cFos, maintains the chondrocytes in a proliferative state (Ref. 2). In order for the chondrocytes to exit the cell cycle and become hypertrophic, Runx2 must be upregulated and Sox9 suppressed. The exit from the cell cycle and apoptosis is necessary for the eventual invasion by osteoblasts and ossification of the bone matrix (Ref. 2).

\section{Osteoblastogenesis}

The transcription factor Runx2 is absolutely necessary for osteoblast differentiation. Runx2 knockout (KO) mice show a total absence of differentiated osteoblasts and, therefore, lack any bone (Refs 18, 19). As Sox9 suppresses Runx2 activity, osteoblast differentiation occurs when Runx2 is upregulated and stabilised. Twist proteins, transcription factors with important roles in embryogenesis, also negatively regulate Runx2, by blocking its DNA binding domain and inhibiting its ability to upregulate osteoblast-specific genes (Ref. 20). Therefore, the inhibition of Twist proteins, specifically Twist-1 and/or Twist-2, is thought to initiate osteoblastogenesis (Ref. 20). Members of the distal-less (Dlx) family of homeobox proteins (Dlx5 and Dlx6) are important for endochondral ossification in the developing appendicular and axial skeleton (Ref. 21). Dlx5 has been shown to act directly upstream of Runx2, by binding its gene promoter, (Ref. 22) and Dlx5/Dlx6 double $\mathrm{KO}$ mice exhibit delayed ossification (Ref. 23). Other homeobox transcription factors are msh homeobox 1 and 2 (Msx1/Msx2), although 
whether their regulation of osteoblast differentiation is positive or negative is still controversial (Ref. 24). Msx1/Msx2 may be necessary for the expression of Runx2, as the Msx1/Msx2 double KO fails to express Runx2 (Ref. 25), although more direct evidence is needed (Fig. 1 summarises major transcriptional regulators of osteoblastogenesis). Wnt signalling has also been implicated in triggering osteoblast differentiation (reviewed in Refs 26, 27). There is a $\beta$-catenin responsive TCF/Lef binding site on the Runx2 gene promoter, and therefore, may upregulate Runx2 expression (Ref. 28). Once upregulated, Runx2 triggers osteoblast differentiation and bone development by binding promoters of osteoblast-specific genes, including Osterix, alkaline phosphatase and Osteocalcin. Once its expression is stabilised, Runx2 inhibits Sox9 transcriptional activity (Ref. 29), further pushing the MSC to the

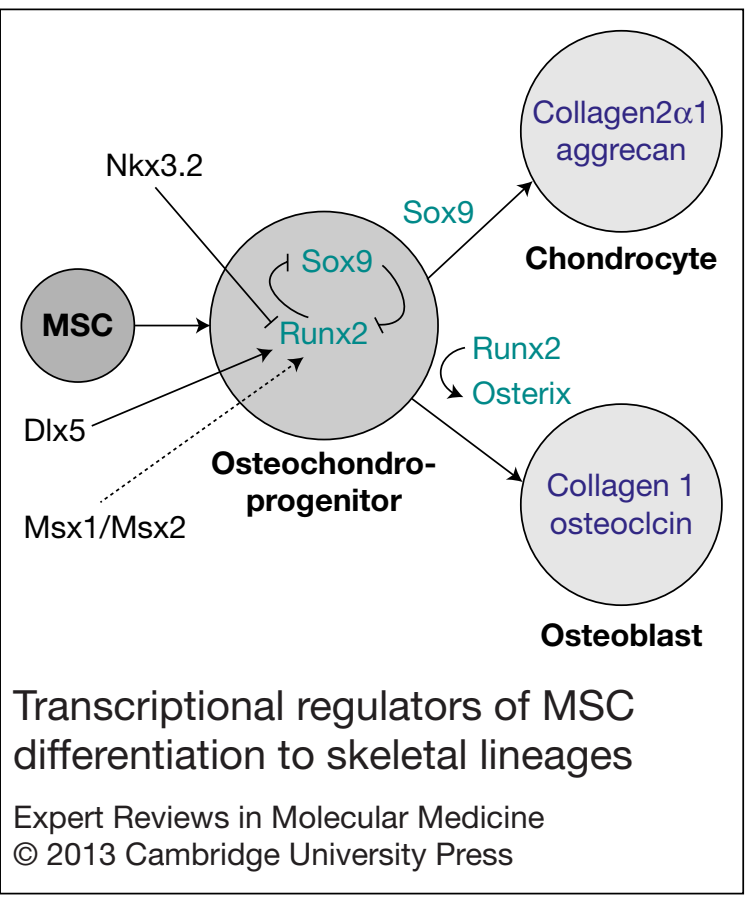

Figure 1. Transcriptional regulators of mesenchymal stem cell (MSC) differentiation to skeletal lineages. Osteochondroprogenitors arise from MSCs and express both Runx2 and Sox9. Multiple transcriptional regulators (including Dlx5, Nkx3.2 and Msx1/Msx2) have been found to modify the expression of Runx2 and Sox9 and, therefore, drive differentiation to chondrocytes or osteoblasts. Supportive references are included in the text. osteoblast lineage. In addition to regulating osteoblast differentiation, Wnt signalling also regulates osteoblast number and function. Postnatal deletion of $\beta$-catenin in Osterix-expressing cells causes osteopenia, however the number of osteoblasts is increased (Ref. 30). Therefore a continued investigation of the role of Wnt signalling in osteoblast function, particularly osteoclast regulation, is needed.

In summary, it appears that the differentiation to chondrocytes may occur by default, when Runx 2 and osteoblast differentiation are suppressed. It is also possible that signals that upregulate Sox 9 are responsible for the suppression of the osteoblast differentiation programme. Figure 2 illustrates these two paths of differentiation.

\section{Cyclic-AMP as a regulator of} osteoblastogenesis and chondrogenesis Although there are master regulators that direct MSC differentiation, various other molecules have been demonstrated to modulate the process. Here, we will focus on those that signal through cyclic-AMP (cAMP). cAMP is a ubiquitous second messenger that is synthesised from ATP by adenylyl cyclases. cAMP levels are regulated by different stimuli, one major effector being G-protein-coupled receptors. These receptors are classified either as stimulatory

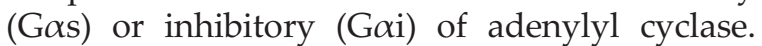
Changing levels of cAMP is translated to the cell through cAMP's action on cyclic nucleotidegated ion channels, on exchange proteins known as Epacs, and on protein kinase A (PKA) (reviewed in Ref. 31). PKA activation further perpetuates the signal by phosphorylating different target proteins. Ultimately, cellular transcription can be modified through the cAMP-dependent transcription factors CREB, cAMP response element modulator (CREM) and ATF-1 (reviewed in Refs 31, 32, 33). cAMP is degraded by phosphodiesterases, which remove a phosphodiester bond and produce AMP. These enzymes play a major role, not only in terminating the signal, but also in regulating the amplitude and duration of the signal (Ref. 34).

The role of Gas signalling in osteoblast differentiation was spurred by the finding that individuals with mutations in the $\mathrm{G} \alpha \mathrm{s}$ gene have a bone phenotype. Inactivating mutations in the Gas gene (GNAS) cause Albright hereditary osteodystrophy (Ref. 35), whereas activating mutations cause fibrous dysplasia of 
Figure 2. Osteoblast versus chondrocyte differentiation. Differentiation to the chondrocyte lineage may occur, by default, with suppression of Runx2 and osteoblastogenesis. Conversely, osteoblast differentiation may be actively suppressed by Sox 9 and/or its regulators.

bone (Ref. 36). To explore its role in bone development, Hsaio et al. created a mouse expressing an engineered $\mathrm{G} \alpha \mathrm{s}$ receptor in osteoblasts and found it to have drastically increased bone mass (Ref. 37). Conversely, complete $\mathrm{KO}$ of $\mathrm{G} \alpha$ s receptors in osteoblasts reduced the number of osteoblasts and impaired bone formation (Refs 38, 39). Although interpretation of these types of experiments is limited, they support a potential role for cAMP signalling in MSC differentiation. In accordance, parathyroid hormone (PTH), a hormone critical for bone development and homoeostasis, signals through a G-protein-coupled receptor (reviewed in Ref. 40), reinforcing the importance of cAMP signalling in skeletogenesis. The PTH receptor binds PTH, as well as PTH-related protein (PTHrP), and is coupled to $\mathrm{G} \alpha$ s as well as $\mathrm{G} \alpha \mathrm{q}$ (Ref. 41). There is a long history of PTH's effect on osteoblast differentiation. Studies in PTH receptor $\mathrm{KO}$ mice show that the signalling is not required for osteoblast differentiation (Ref. 42), however PTH has been demonstrated to enhance osteoblast differentiation (reviewed in Ref. 43). Recently, it was found that PTH interacts with canonical Wnt signalling and FGF-2 signalling and that this interaction enhances osteoblast differentiation (reviewed in Ref. 44). Also, PTH signalling was found to interact with bone morphogenetic protein signalling, which additionally enhances osteoblast differentiation (Refs 45, 46, 47, 48).

The direct effect of cAMP on chondrogenesis has not been fully investigated. In one study, treatment of rabbit chondrocyte cultures with cAMP analogues was found to suppress terminal differentiation and hypertrophy of chondrocytes. Similarly to osteoblasts, PTH is not required for chondrocyte differentiation (Ref. 42). However, PTH treatment of C3H10T1/2 cells can enhance the early stages of chondrocyte differentiation while suppressing chondrocyte maturation (Ref. 49). In addition, limb explants from PTHrP $\mathrm{KO}$ mice displayed accelerated chondrocyte maturation (Ref. 50).

cAMP effect on transcriptional regulators of osteoblastogenesis

Studies have shown that increasing intracellular levels of cAMP in cell lines or in primary mouse and human MSCs, through cAMP analogues or forskolin (a direct adenylyl cyclase activator), has a positive effect on Runx2 expression (Refs 51, 52, 53, 54). In addition, activation of receptors that signal through cAMP increases Runx2 expression in MSC cell lines and in primary rodent MSCs. These include the PTH receptor (Ref. 55) and the A2B adenosine receptor (Refs 54, 56). This increase in expression may be owing to cAMP activation of 
CREB, as the Runx2 promoter contains a functional CREB domain (Ref. 57). Another likely effect of cAMP is its modulation of Runx2 activity. Runx2 has putative PKA phosphorylation sites in its activation domain, and treatment with a PKA-specific cAMP analogue increases its phosphorylation (Refs 58, 59) and activation of the collagenase- 3 promoter (Ref. 58) and the matrix metalloproteinase-13 promoter (Ref. 59). The role that PKA phosphorylation of Runx2 has in vivo during osteoblast differentiation remains to be determined.

As mentioned earlier, Osterix is another transcription factor essential for osteoblast differentiation and bone development. As Runx2 KO mice do not express Osterix (Refs 18, 19) but Osterix KO mice do express Runx2, it was deduced that Osterix is downstream of Runx2 (Ref. 60). Analysis of the Osterix gene promoter identified a Runx2 binding site, and Runx2 was reported to upregulate Osterix expression (Ref. 61). A putative CREB site was also found in this gene promoter, suggesting cAMP may be able to regulate Osterix expression (Ref. 61). In support of this, treatment of cells lines or primary mouse MSCs with cAMP analogues increases Osterix expression (Refs 51, 54, 62). However, exposure to relatively high cAMP (1 mM) inhibits its expression in UM-106-01 cell line or in mouse primary osteoblasts (Ref. 63). Similarly to Runx2 expression, Gas receptor activation causes an increase in Osterix expression (Ref. 54), the mechanism of which remains to be demonstrated.

Dlx5, an upregulator of Runx2 (Ref. 22), is phosphorylated by PKA, which increases Dlx5 protein levels by augmenting its stability. PKA signalling also increases Dlx5 transcriptional activity. Therefore, PKA signalling enhances Dlx5-induced osteoblast differentiation (Ref. 64).

Of note, some have reported a negative effect of cAMP on osteoblast differentiation. Yang et al. found that the PKA inhibitor, PKI, increased the expression of Runx2 in a human MSC-derived cell line whereas forskolin, 3-isobutyl-1methylxantine and a cAMP analogue decreased the expression of osteopontin (Ref. 65). Koh et al. found forskolin to decrease osteocalcin expression in MC3T3-E1 cells. Forskolin also decreased the number of mineralised nodules formed by rat primary calvarial cells (Ref. 66). Tintut et al. reported that treatment of MC3T3-E1 cells with forskolin decreased the activity of alkaline phosphatase and inhibited mineralisation, as well as decreased the expression of alkaline phosphatase, bone sialoprotein, osteocalcin and osteopontin (Ref. 67). The discrepancy in reported effects of cAMP on osteoblastogenesis may be due, in part, to the relatively high concentration of forskolin or cAMP analogue used (10-100 $\mu \mathrm{M})$. For instance, Turksen et al. found that treatment with $10 \mu \mathrm{M}$ of forskolin inhibited osteoblast differentiation whereas $1 \mathrm{~nm}$ increased it (Ref. 68).

\section{cAMP effect on Sox9 and chondrogenesis}

Multiple studies have suggested synergism between cAMP signalling and Sox9 expression. The Sox9 promoter contains a CRE site (Ref. 69) and, using a Sox9 gene promoter reporter construct in various MSC cell lines, it was reported that binding of this site by CREB increases Sox9 promoter activity (Ref. 70).

Sox9 interacts with CREB binding protein (CBP) and p300 to increase its transcriptional activity. Using a Col2 $\alpha 1$ gene promoter reporter construct in a chondrocyte cell line, as well as a Gal4-Sox9 fusion protein, it was reported that co-transfection with CBP and/or p300 increased Sox9 activity as a transcriptional activator (Ref. 71).

A yeast two-hybrid screen of Sox9 binding partners identified the PKA catalytic subunit as interacting with Sox9 (Ref. 72). This prompted the investigation of PKA-dependent Sox9 phosphorylation and two serine residues were found to be phosphorylated (Ref. 72). Phosphorylation of these sites by PKA increased Sox9 activation of the Col2 $\alpha 1$ gene promoter (Ref. 72). Zhao et al. investigated this finding further by mutating the serine residues. The absence of PKA phosphorylation partially reversed PKA enhancement of Sox9 activity, suggesting that PKA augmented Sox9 activity through multiple mechanisms. The investigators found an interaction between Sox9 and CBP in chondrogenic differentiating $\mathrm{C} 3 \mathrm{H} 10 \mathrm{~T} 1 / 2$ cells by using co-immunoprecipitation, and this interaction increased Sox9 transcriptional activity. The importance of this interaction was confirmed by mutating a CREB site in the region of the Sox9 interaction, which reversed the effect (Ref. 73). Therefore, cAMP signalling, by means of PKA, enhances Sox9 expression and transcriptional activity through direct phosphorylation and activation of CREB. In addition to findings in a cell line, differentiation 
of chick limb bud chondrocytes in the presence of the PKA inhibitor H89 blocked chondrogenesis. Yoon et al. found PKA to promote chondrogenesis by downregulating N-Cadherin through a PKC-dependent pathway (Ref. 74).

Taken together, it appears that cAMP signalling is capable of upregulating Runx2, a master regulator of osteoblasts, and Sox9, a master regulator of chondrocytes. Since Sox9 is an inhibitor of Runx2 expression, it is possible that the sum effect of cAMP on lineage determination depends on its concentration, cellular localisation and a possible regulatory loop.

\section{Adenosine receptors}

Adenosine is a regulatory metabolite and its receptors are comprised of a family of seven transmembrane domain $G$ protein-coupled receptors. A1 and $\mathrm{A} 3$ adenosine receptors (A1AR and A3AR) are coupled to Gai and are, therefore, adenylyl cyclase inhibiting, whereas $\mathrm{A} 2 \mathrm{~A}$ and $\mathrm{A} 2 \mathrm{~B}$ adenosine receptors (A2AAR and A2BAR) are coupled to $G \alpha s$, which stimulates adenylyl cyclase and produces a cAMP signal. The A2BAR may also be coupled to G $\alpha q$, which activates phospholipase C (Ref. 75). A2BAR has a relatively low affinity for adenosine. High extracellular concentrations of adenosine can be achieved during cell injury or stress (Ref. 76). A2AAR and A2BARs are widely expressed to varying degrees, with high expression in the vasculature (Ref. 77). Our laboratory and others have shown that A2BAR expression is inducible under stress conditions such as inflammation and hypoxia (reviewed in Ref. 78). A2AAR and A2BAR are expressed in MSCs (Refs 56, 79), and interestingly, high expression of the A2AAR and $\mathrm{A} 2 \mathrm{BAR}$ receptors can be found in cartilage (Ref. 80).

\section{Adenosine receptors and osteoblast and chondrocyte differentiation}

Only recently has the role of adenosine receptors in osteoblast differentiation been examined. In a broad sense, purinergic signalling was looked at in the context of bone, and ATP receptors (P2X and $\mathrm{P} 2 \mathrm{Y}$ ) were found to promote differentiation and proliferation in an osteoblast cell line (MC3T3-E1) (Ref. 81). It was found that after bone injury and when exposed to hypoxic conditions, rat osteoblasts secrete ATP in the high nм to $\mu \mathrm{m}$ range (Ref. 82). This extracellular ATP is available for catabolism to adenosine by ectonucleotidases, which are expressed on osteoblasts (Ref. 79). In accordance, it was recently demonstrated that genetic ablation of the ectonucleotidase CD73 results in osteopenia and decreased osteoblast differentiation in mice (Ref. 83).

In vitro studies found adenosine receptors to be expressed in both human (Ref. 79) and rodent MSCs (Ref. 56). Based on its expression and measurements of cAMP levels after agonist treatments of rat MSCs, Gharibi et al. concluded that the A2BAR is the dominant receptor, relative to other adenosine receptors, and that its expression increases during osteoblast differentiation (Ref. 56). In human MSCs, A2BAR activation increased osteoblast differentiation, as determined by an increase in alkaline phosphatase activity (Ref. 79). Similarly, in rat MSCs, activation of the A2BAR increased Runx2 and alkaline phosphatase expression, as well as the number of mineralised nodules (Ref. 56). We found bone marrow-derived MSCs from A2BAR $\mathrm{KO}$ mice to have decreased osteoblast differentiating potential, with diminished expression of Runx2 and Osterix. Activation of the A2BAR with pharmacological agonists increased the expression of these transcription factors, as well as caused an increase in the number of mineralised nodules. Treatment with a cAMP analogue also increased the expression of Osterix, suggesting that cAMP may be the mechanism of action for A2BAR effect on differentiation. In addition, the A2BAR KO mouse had mild osteopenia, and a delayed or impaired bone fracture healing response (Ref. 54).

There have been few reports on the effect of the cAMP inhibitory adenosine receptors, A1AR and $\mathrm{A} 3 \mathrm{AR}$ on osteoblast differentiation. Overexpression of the A1AR in an osteoblast precursor cell line led to inhibition of osteoblast differentiation, and instead promoted differentiation to adipocytes (Ref. 84). However, treatment of human MSCs with the A1AR agonist, N6-cyclopentyladenosine, caused an increase in osteoblast differentiation (Ref. 79). In rat MSCs, very little A3AR was found (Ref. 56) and in human MSCs, treatment with the A3AR agonist, 1-Deoxy-1-[6-[[(3-iodophenyl)methyll] amino]-9Hpurin-9-yl]- $N$-methyl- $\beta$-D-ribofuranuronamide, had no effect on osteoblast differentiation (Ref. 79).

A role for adenosine receptors in chondrocyte differentiation has not yet been examined and/ 
or reported. However, as A1- and A2-type ARs are known to be expressed in MSCs (Refs 56, 79), and given the published influence of cAMP on Sox9 (as described above), it is likely that a role for these receptors on the differentiation of this lineage will be found.

\section{Inflammation and osteoblast and chondrocyte differentiation: implication for adenosine receptors}

Inflammation plays a significant role in bone development (reviewed in Ref. 85) and regeneration (reviewed in Ref. 86). Although adenosine receptor signalling through cAMP has the potential to directly influence osteo- or chondrogenesis, cAMP signalling through these receptors can also affect the level of inflammatory cytokines systemically or at the cellular level (reviewed in Ref. 87). Here, we will focus on a brief survey of the effects of inflammatory processes on bone cell differentiation, followed by a summary of A2type adenosine receptors effects on inflammation and its potential influence on bone cell lineages.

\section{The effect of inflammation on osteoblast differentiation}

The effect of inflammation on the skeletal system, including osteoblast differentiation, has been well studied, particularly the effects of tumour necrosis factor- $\alpha$ (TNF- $\alpha$ ) (reviewed in Ref. 88). Experiments by Gilbert et al. in both fetal calvarial cells and the osteoblast precursor cell line MC3T3-E1 show that TNF- $\alpha$ inhibits osteoblast differentiation, specifically at the early stage of lineage commitment (Ref. 89). Further experimentation showed this inhibition to be associated with downregulation of Runx2 transcription and a subsequent decrease in nuclear Runx2 (Ref. 90). Although these authors did not find NFKB signalling to be involved, Huang et al. found that inhibition of $N F \kappa B$ signalling by overexpression of I $\mathrm{KB}$ in ST2 cells, a MSC line, abolished the inhibitory effect of TNF- $\alpha$ on Runx 2 gene expression (Ref. 91). The inhibitory effect of TNF- $\alpha$ on mRNA expression and osteoblast differentiation was confirmed in primary mouse MSCs. Here, Lacey et al. also found interleukin-1 $\beta$ (IL-1 $\beta$ ) to have a similar effect (Ref. 92).

These studies on mRNA expression were complimented by Kaneki et al. who found
TNF- $\alpha$ to promote Runx 2 protein degradation in $\mathrm{C} 2 \mathrm{C} 12$ and $2 \mathrm{~T} 3$ osteoblast precursor cells by upregulating the E3 ligases Smurf1 and Smurf2 (Ref. 93). In order to examine this phenomenon in primary MSCs, Zhao et al. isolated an MSCenriched fraction from the bone marrow of TNF- $\alpha$ overexpressing mice. In these cells they found upregulation of the E3 ligase Wwp1 relative to cells from wildtype (WT) mice. Further experimentation found Wwp1 to be upregulated by TNF- $\alpha$ and responsible for inhibiting osteoblast differentiation. However, in these primary cells, the inhibition was caused by the degradation of JunB, a promoter of osteoblast differentiation, rather than affecting Runx2 directly (Ref. 94).

In addition to their studies on Runx2, Lu et al. found TNF- $\alpha$ treatment to inhibit Osterix mRNA expression, and claim this to be a direct effect of TNF- $\alpha$ signalling through mitogen activated (MEK) and inhibition of the Osterix gene promoter (Ref. 95). In addition, they found TNF- $\alpha$-stimulated binding of paired mesoderm homeobox protein 1(Prx1), causing inhibition of Osterix gene promoter activity and transcription in MC3T3 and C3H10T1/2 cells (Ref. 96). Interestingly, Prx1 is a developmental regulator of skeletogenesis that was previously thought to be silenced after embryogenesis (Ref. 96).

Other regulators of osteoblast differentiation are affected by TNF- $\alpha$. In culture, Msx2 has been shown to inhibit osteoblast differentiation (Ref. 97). Treatment of $\mathrm{C} 2 \mathrm{C} 12$ or fetal calvarial cells with TNF- $\alpha$ caused increased expression of Msx2 and reduced expression of alkaline phosphatase. This effect was independent of Runx2, as the phenomenon was maintained in Runx2 null cells, and overexpression of dominant negative I $\kappa$ B showed NFkB signalling to be involved. To model inflammation induced by wear of artificial limbs, macrophages were activated with titanium particles and their media, enriched in inflammatory cytokines, was used to treat MC3T3-E1 cells. Treatment with this conditioned media or TNF- $\alpha$ inhibited Runx2 expression and osteoblast differentiation and this was attributed to $\mathrm{NF} \kappa \mathrm{B}$ activation and increased expression of sclerostin, an inhibitor of the Wnt pathway (Ref. 98).

\section{The effect of inflammation on chondrocyte} differentiation

Relative to osteoblastogenesis, little is known about the effect of inflammation on chondrocyte 
differentiation. IL-1 and TNF- $\alpha$ decreased Sox9 mRNA and protein expression in a chondrocytic cell line, MC615, and in primary mouse chondrocytes through the NFkB signalling pathway (Refs 99, 100). However, in these studies differentiation was not directly addressed. To study chondrocyte differentiation, Nakajima et al. differentiated a chondrocyte progenitor cell line, ATDC5, in the presence of IL-6. IL-6 decreased collagen $2 \alpha 1$ and collagen 10 mRNA expression in a dose-dependent manner and inhibited the formation of cartilage nodules (Ref. 101). Wehling et al. differentiated human MSCs to chondrocytes in the presence of either IL-1 or TNF- $\alpha$. They found that both cytokines decreased the size of the cartilage pellet and lowered the amount of

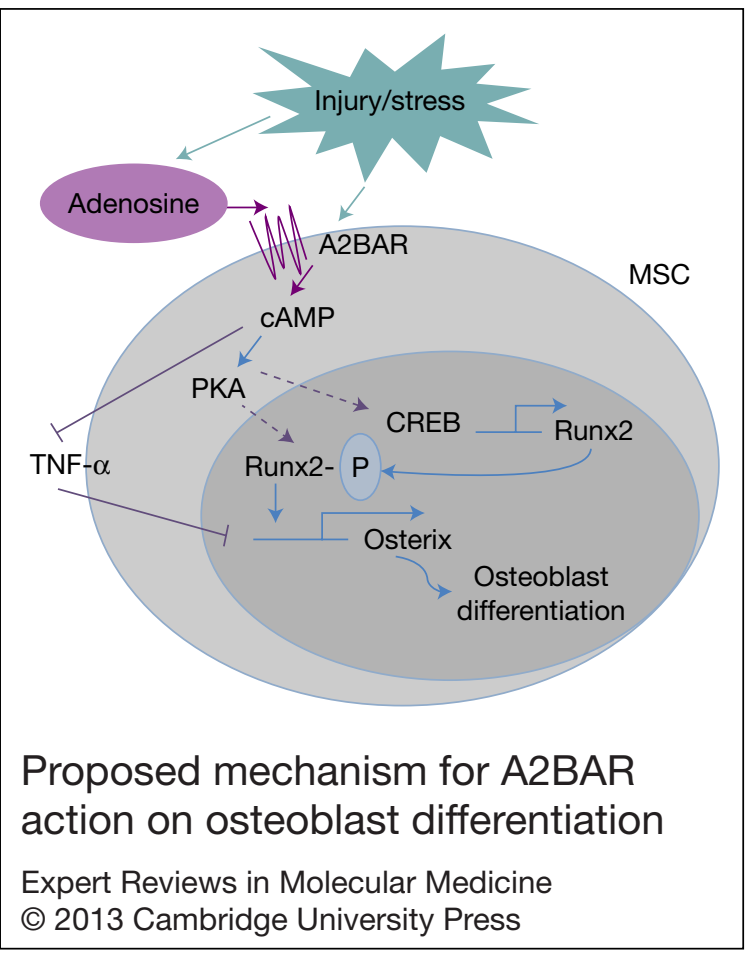

Figure 3. Proposed mechanism for A2BAR action on osteoblast differentiation. Activation of the mesenchymal stem cell A2BAR triggers CAMP signalling, which may modulate the expression and/or activity of a key osteoblast transcription factor, Runx2, and promote osteoblast differentiation. A2BAR activation also decreases tumour necrosis factor- $\alpha$ level, which has an inhibitory effect on osteoblast differentiation. glycosaminoglycan accumulation. IL-1 treatment decreased the expression of Col2 $\alpha 1$ and aggrecan mRNA (Ref. 102).

Taken together, it is then quite possible that adenosine receptor modulation of inflammatory cytokines affects directly or indirectly the process of MSC differentiation into bone cell lineages. Figure 3 illustrates a proposed mechanism of A2BAR effects on osteoblast differentiation involving CREB and/or TNF- $\alpha$.

\section{Adenosine receptors and inflammation}

One of the principal attributes of adenosine and its receptors is its pro- or anti-inflammatory effects, most of which are mediated via cAMP signalling. The concentration of our laboratory has been the A2BAR, and thus will be the focus of this section. Both pro- and anti-inflammatory effects of the A2BAR have been described, and these depend on the cell type and stimulus. In addition, these effects have been ascribed either protective or deleterious roles, depending on the context, e.g. chronic versus acute pathology (reviewed in Ref. 103). Complete KO of the A2BAR gene results in a slight systemic inflammation, as $\mathrm{KO}$ animals have elevated plasma levels of TNF- $\alpha$ at baseline, and elevated levels of TNF- $\alpha$ and IL-6 expression in macrophages (Ref. 104). These differences in cytokine levels are exacerbated upon stress or injury. Treatment of A2BAR KO mice with lipopolysaccharide causes an exceptional increase in TNF- $\alpha$ and IL-6 plasma levels and in macrophage expression, relative to WT mice (Ref. 104).

The ability of the A2BAR to dampen inflammation in response to stimuli highlights its protective role during stress or injury. The importance of this role is confirmed by findings that the expression of the receptor is also induced by these stimuli. Treatment of vascular smooth muscle cells with TNF- $\alpha$ causes an upregulation of A2BAR expression. Further, this increase was shown to be mediated by NADPH oxidase 4 (Nox4) signalling (Ref. 105).

As NF $\kappa B$ has an important role in the regulation of cellular inflammation it has been a target of investigation in relation to A2BAR signalling. Recently, we found the A2BAR to directly bind p105, an inhibitor of $\mathrm{NF \kappa B}$, stabilising it and preventing its degradation (Ref. 106). This mechanism helps explain the inflammatory phenotype in the A2BAR $\mathrm{KO}$ mice and the 
ability of the A2BAR to downregulate inflammation.

Considering the above described effects of TNF$\alpha$, IL- 1 and IL- 6 on bone cell differentiation, it is possible that some of the A2BAR's recently described protective effect in a mouse bone injury model (Ref. 54) is related to changes in cytokine levels.

\section{Adenosine receptors as therapeutics for osteoporosis, skeletal injury and arthritis}

A role for adenosine receptors in osteoporosis is gaining increasing momentum. In mouse bone marrow-derived cells, antagonism of the A1AR with 8-cyclopentyl-1,3-dipropylxanthine inhibited the differentiation of osteoclasts (bone reabsorbing cells) (Ref. 107). Also in these cells, treatment with an A2AAR agonist, 4-[2-[[6Amino-9-( $N$-ethyl- $\beta$-D-ribofuranuronamidosyl)9H-purin-2-yl]amino]ethyl]benzene propanoic acid hydrochloride (CGS21680), inhibited the differentiation of osteoclasts, as well as inhibited their activity. In addition, the A2AAR KO mouse has an increased number of osteoclasts, as well as reduced bone volume (Ref. 108). Similarly, A2BAR KO mice display a mild reduction in bone density (Ref. 54). In our study, we also found that $\mathrm{A} 2 \mathrm{BAR} \mathrm{KO}$ mice have changes in bone fracture healing with decreased callus bone formation and an apparent delay in healing (Ref. 54). Although the anti-inflammatory effects of adenosine likely have an important role in improving skeletal injury repair, we contend that adenosine, through the A2BAR, may also improve healing by promoting osteoblast differentiation (Ref. 54). Therefore, agonism of the A2-type ARs may be useful as a therapeutic for osteoporosis and bone injury.

As caffeine is an antagonist of adenosine receptors, its effects on bone may be relevant here. In epidemiological studies of risk factors for osteoporosis in humans, caffeine was either found to be negatively associated with (Refs 109,110 ) or not associated with bone mineral density (Refs 111, 112, 113, 114). It is possible that effects of caffeine are confounded by factors such as age, oestrogen levels and calcium intake. In a study of over 3000 individuals using the Framingham Cohort, it was found that caffeine intake was associated with a higher relative risk of hip fracture (Ref. 115). In experimental animals, caffeine has been shown to inhibit bone formation. When demineralised bone particles were implanted subcutaneously, rats that were treated with caffeine had decreased chondrogenesis and decreased mineralisation (Ref. 116), suggesting that caffeine impairs new endochondral bone formation by inhibiting the proliferation and differentiation of chondroprogenitor cells. In addition, chick osteoblasts treated with caffeine had decreased collagen expression and alkaline phosphatase activity, resulting in reduced matrix formation (Ref. 117). Finally, differentiation of osteoclasts from mouse bone marrow-derived cells was enhanced with caffeine treatment (Ref. 118). Whether any or all of these negative effects of caffeine on bone formation and maintenance is solely because of antagonism of adenosine receptors has not been determined.

Adenosine receptors have been found to be protective against a variety of injuries, including but not limited to cardiovascular (reviewed in Ref. 119), kidney (reviewed in Ref. 120), lung (reviewed in Ref. 121) and gastrointestinal (reviewed in Ref. 122). As arthritis is an inflammatory disease of the joints, adenosine receptors have been investigated in the context of this disease. Direct infusion of adenosine into the joint in a rat arthritis model reduced the pathogenesis of the disease (Ref. 123). Methotrexate is a drug commonly used for the treatment of rheumatoid arthritis and ameliorates the condition by decreasing the inflammatory response in the joints. At least part of its inflammatory action is attributed to its ability to increase adenosine release in the joints (Ref. 124). Currently, the principal receptor to be implicated is the A3AR. An A3AR receptor agonist, CF-101, has been undergoing clinical trials for the treatment of rheumatoid arthritis; however, the improvement in rheumatoid arthritis has not yet reached statistical significance (Ref. 125).

\section{Conclusions}

There are a multitude of signalling molecules and pathways that converge during chondrocyte and osteoblast differentiation. The coordination of the spatial and temporal pattern of these molecules is necessary for correct bone formation. Through exploration of the downstream signalling of G-protein-coupled receptors, cAMP was found to be an important component of the signalling pathways needed for full differentiation of MSCs along the skeletal lineage. Continued investigation into the 
contribution of these receptors, adenosine receptors among them, to bone homoeostasis and regeneration could lead to important discoveries with clinically therapeutic implications.

\section{References}

1 Olsen, B.R., Reginato, A.M. and Wang, W. (2000) Annual Review of Cell and Developmental Biology 16, 191-220

2 Hartmann, C. (2009) Current Opinion in Genetics \& Development 19, 437-443

3 Schindeler, A. et al. (2008) Seminars in Cell \& Developmental Biology 19, 459-466

4 Baldridge, D. et al. (2010) Annual Review of Genomics and Human Genetics 11, 189-217

5 DeLise, A.M., Fischer, L. and Tuan, R.S. (2000) Osteoarthritis and Cartilage 8, 309-334

6 Hall, B.K. and Miyake, T. (2000) Bioessays 22, 138-147

7 Enomoto, H. et al. (2000) Journal of Biological Chemistry 275, 8695-8702

8 Hill, T.P. et al. (2005) Developmental Cell 8, 727-738

9 Day, T.F. et al. (2005) Developmental Cell 8, 739-750

$10 \mathrm{Hu}, \mathrm{H}$. et al. (2005) Development 132, 49-60

11 Foster, J.W. et al. (1994) Nature 372, 525-530

12 Wagner, T. et al. (1994) Cell 79, 1111-1120

13 Augello, A. and De Bari, C. (2010) Human Gene Therapy 21, 1226-1238

14 Smith, N. et al. (2005) Journal of Cellular Physiology 203, 133-143

15 Zhou, G. et al. (2006) Proceedings of the National Academy of Sciences of the United States of America 103, 19004-19009

16 Cheng, A. and Genever, P.G. (2010) Journal of Bone and Mineral Research 25, 2680-2689

17 Lengner, C.J. et al. (2005) Journal of Biological Chemistry 280, 15872-15879

18 Otto, F. et al. (1997) Cell 89, 765-771

19 Komori, T. et al. (1997) Cell 89, 755-764

20 Bialek, P. et al. (2004) Developmental Cell 6, 423-435

21 Kraus, P. and Lufkin, T. (2006) American Journal of Medical Genetics. Part A 140, 1366-1374

22 Lee, M.H. et al. (2005) Journal of Biological Chemistry 280, 35579-35587

23 Robledo, R.F. et al. (2002) Genes \& Development 16, 1089-1101

24 Komori, T. (2006) Journal of Cellular Biochemistry 99, 1233-1239

25 Han, J. et al. (2007) Mechanisms of Development $124,729-745$

26 Lin, G.L. and Hankenson, K.D. (2011) Journal of Cellular Biochemistry 112, 3491-3501
27 Milat, F. and Ng, K.W. (2009) Molecular and

Cellular Endocrinology 310, 52-62

28 Dong, Y.F. et al. (2006) Journal of Cellular Physiology 208, 77-86

29 Topol, L. et al. (2009) Journal of Biological Chemistry 284, 3323-3333

30 Chen, J. and Long, F. (2012) Journal of Bone and Mineral Research doi: [10.1002/jbmr.1834] [epub ahead of print]

31 Sands, W.A. and Palmer, T.M. (2008) Cellular Signalling 20, 460-466

32 Mayr, B. and Montminy, M. (2001) Nature Reviews. Molecular Cell Biology 2, 599-609

33 Lee, K.A. and Masson, N. (1993) Biochimica et Biophysica Acta 1174, 221-233

34 Beavo, J.A. (1995) Physiological Reviews 75, 725-748

35 Wilson, L.C. and Hall, C.M. (2002) Seminars in Musculoskeletal Radiology 6, 273-283

36 Riminucci, M. et al. (2010) Journal of Molecular Endocrinology 45, 355-364

37 Hsiao, E.C. et al. (2008) Proceedings of the National Academy of Sciences of the United States of America 105, 1209-1214

38 Sakamoto, A. et al. (2005) Journal of Biological Chemistry 280, 21369-21375

$39 \mathrm{Wu}$, J.Y. et al. (2011) Journal of Clinical Investigation 121, 3492-3504

40 Datta, N.S. and Abou-Samra, A.B. (2009) Cellular Signalling 21, 1245-1254

41 Abou-Samra, A.B. et al. (1992) Proceedings of the National Academy of Sciences of the United States of America 89, 2732-2736

42 Lanske, B. et al. (1996) Science 273, 663-666

43 Jilka, R.L. (2007) Bone 40, 1434-1446

44 Fei, Y. and Hurley, M.M. (2012) Journal of Cellular Physiology 227, 3539-3545

$45 \mathrm{Yu}, \mathrm{B}$. et al. (2012) Journal of Bone and Mineral Research 27, 2001-2014

46 Takase, H. et al. (2009) Hormone and Metabolic Research 41, 861-865

47 Nakao, Y. et al. (2009) Bone 44, 872-877

48 Chan, G.K. et al. (2003) Endocrinology 144, 5511-5520

49 Hollnagel, A., Ahrens, M. and Gross, G. (1997) Journal of Bone and Mineral Research 12, 1993-2004

50 Vortkamp, A. et al. (1996) Science 273, 613-622

51 Ghayor, C. et al. (2009) Biochemical and Biophysical Research Communications 381, 247-252

52 Siddappa, R. et al. (2008) Proceedings of the National Academy of Sciences of the United States of America 105, 7281-7286

53 Lo, K.W. et al. (2012) Journal of Tissue Engineering and Regenerative Medicine 6, 40-48 
54 Carroll, S.H. et al. (2012) Journal of Biological Chemistry 287, 15718-15727

55 Krishnan, V. et al. (2003) Molecular Endocrinology $17,423-435$

56 Gharibi, B. et al. (2011) Journal of Bone and Mineral Research 26, 2112-2124

57 Xiao, Z.S. et al. (2001) Journal of Cellular Biochemistry 82, 647-659

58 Selvamurugan, N. et al. (2000) Journal of Biological Chemistry 275, 5037-5042

59 Selvamurugan, N. et al. (2009) FEBS Letters 583, 1141-1146

60 Nakashima, K. et al. (2002) Cell 108, 17-29

61 Nishio, Y. et al. (2006) Gene 372, 62-70

62 Wang, B.L. et al. (2006) Journal of Endocrinological Investigation 29, 101-108

63 Hong, S.H. et al. (2009) Journal of Molecular Endocrinology 43, 197-207

64 Han, Y. et al. (2011) Biochemical and Biophysical Research Communications 407, 461-465

65 Yang, D.C. et al. (2008) PLoS One 3, e1540

66 Koh, A.J. et al. (1999) Endocrinology 140, 3154-3162

67 Tintut, Y. et al. (1999) Journal of Biological Chemistry 274, 28875-28879

68 Turksen, K. et al. (1990) Journal of Cellular Physiology 142, 61-69

69 Kanai, Y. and Koopman, P. (1999) Human Molecular Genetics 8, 691-696

70 Piera-Velazquez, S. et al. (2007) Experimental Cell Research 313, 1069-1079

71 Tsuda, M. et al. (2003) Journal of Biological Chemistry 278, 27224-27229

72 Huang, W. et al. (2000) Molecular and Cellular Biology 20, 4149-4158

73 Zhao, L., Li, G. and Zhou, G.Q. (2009) Journal of Bone and Mineral Research 24, 826-836

74 Yoon, Y.M. et al. (2000) Journal of Bone and Mineral Research 15, 2197-2205

75 Fredholm, B.B. et al. (2001) Pharmacological Reviews 53, 527-552

76 Fredholm, B.B. (2007) Cell Death and Differentiation 14, 1315-1323

77 St Hilaire, C. et al. (2009) Journal of Cellular Physiology 218, 35-44

78 Hasko, G. et al. (2009) Trends in Immunology 30, 263-270

79 Costa, M.A. et al. (2011) J Journal of Cellular Physiology 226, 1353-1366

80 Koolpe, M., Pearson, D. and Benton, H.P. (1999) Arthritis and Rheumatism 42, 258-267

81 Shimegi, S. (1996) Calcified Tissue International 58, 109-113

82 Orriss, I.R. et al. (2006) Bone 39, 300-309
83 Takedachi, M. et al. (2011) Journal of Cellular Physiology 227, 2262-2631

84 Gharibi, B. et al. (2012) International Journal of Obesity 36, 397-406

85 De Benedetti, F. (2009) Hormone Research 72(Suppl 1), 26-29

86 Mountziaris, P.M. and Mikos, A.G. (2008) Tissue Engineering Part B: Reviews 14, 179-186

87 Blackburn, M.R. et al. (2009) Handbook of Experimental Pharmacology 195, 215-269

88 Nanes, M.S. (2003) Gene 321, 1-15

89 Gilbert, L. et al. (2000) Endocrinology 141, 3956-3964

90 Gilbert, L. et al. (2002) Journal of Biological Chemistry 277, 2695-2701

91 Huang, H. et al. (2011) Cell Proliferation 44, 420-427

92 Lacey, D.C. et al. (2009) Osteoarthritis and Cartilage 17, 735-742

93 Kaneki, H. et al. (2006) Journal of Biological Chemistry 281, 4326-4333

94 Zhao, L. et al. (2011) Stem Cells 29, 1601-1610

$95 \mathrm{Lu}$, X. et al. (2006) Journal of Biological Chemistry $281,6297-6306$

$96 \mathrm{Lu}$, X. et al. (2011) Journal of Bone and Mineral Research 26, 209-219

97 Lee, H.L. et al. (2010) Experimental \& Molecular Medicine 42, 437-445

98 Lee, S.S. et al. (2012) Biomaterials 33, 4251-4263

99 Murakami, S., Lefebvre, V. and de Crombrugghe, B. (2000) Journal of Biological Chemistry 275, 3687-3692

100 Sitcheran, R., Cogswell, P.C. and Baldwin, A.S., Jr (2003) Genes \& Development 17, 2368-2373

101 Nakajima, S. et al. (2009) Cytokine 47, 91-97

102 Wehling, N. et al. (2009) Arthritis and Rheumatism 60, 801-812

103 Johnston-Cox, H.A., Koupenova, M. and Ravid, K. (2012) Arteriosclerosis, Thrombosis, and Vascular Biology 32, 870-878

104 Yang, D. et al. (2006) Journal of Clinical Investigation 116, 1913-1923

105 St Hilaire, C. et al. (2008) Biochemical and Biophysical Research Communications 375, 292-296

106 Sun, Y. et al. (2012) Journal of Cell Science 125, 4507-4517

107 He, W. and Cronstein, B.N. (2012) Purinergic Signal $8,327-337$

108 Mediero, A. et al. (2012) American Journal of Pathology 180, 775-786

109 Rapuri, P.B. et al. (2001) American Journal of Clinical Nutrition 74, 694-700

110 Ilich, J.Z. et al. (2002) Journal of the American College of Nutrition 21, 536-544 
111 Wetmore, C.M. et al. (2008) Osteoporosis International 19, 519-527

112 Waugh, E.J. et al. (2009) Osteoporosis International 20, $1-21$

113 Hannan, M.T. et al. (2000) Journal of Bone and Mineral Research 15, 710-720

114 Conlisk, A.J. and Galuska, D.A. (2000) Preventive Medicine 31, 562-568

115 Kiel, D.P. et al. (1990) American Journal of Epidemiology 132, 675-684

116 Barone, L.M. et al. (1993) Journal of Cellular Biochemistry 52, 171-182

117 Tassinari, M.S. et al. (1991) Journal of Bone and Mineral Research 6, 1029-1036

118 Liu, S.H. et al. (2011) Journal of Orthopaedic Research 29, 954-960
119 Riksen, N.P. and Rongen, G.A. (2012) Expert Review of Clinical Pharmacology 5, 199-218

120 Bauerle, J.D. et al. (2011) Journal of the American Society of Nephrology 22, 14-20

121 Eckle, T., Koeppen, M. and Eltzschig, H.K. (2009) Physiology (Bethesda) 24, 298-306

122 Colgan, S.P. and Eltzschig, H.K. (2012) Annual Review of Physiology 74, 153-175

123 Green, P.G. et al. (1991) Proceedings of the National Academy of Sciences of the United States of America 88, 4162-4165

124 Cronstein, B.N., Naime, D. and Ostad, E. (1993) Journal of Clinical Investigation 92, 2675-2682

125 Silverman, M.H. et al. (2008) Journal of Rheumatology 35, 41-48

\section{Features associated with this article}

\section{Figures}

Figure 1. Transcriptional regulators of mesenchymal stem cell (MSC) differentiation to skeletal lineages. Figure 2. Osteoblast versus chondrocyte differentiation.

Figure 3. Proposed mechanism for A2BAR action on osteoblast differentiation.

\section{Citation details for this article}

Shannon H. Carroll and Katya Ravid (2013) Differentiation of mesenchymal stem cells to osteoblasts and chondrocytes: a focus on adenosine receptors. Expert Rev. Mol. Med. Vol. 15, e1, February 2013, doi:10.1017/erm.2013.2 